\title{
Role of Src homology 2 domain-mediated PTK signaling in mouse zygotic development
}

\author{
Li Meng, JinPing Luo, Chunhua Li and William H Kinsey \\ Center for Reproductive Sciences and Department of Anatomy and Cell Biology, University of Kansas Medical Center, \\ 3901 Rainbow Boulevard, Kansas City, Kansas 66160, USA
}

Correspondence should be addressed to W H Kinsey; Email: wkinsey@kumc.edu

\begin{abstract}
Fyn and other Src-family kinases play an essential role at several steps during egg activation following fertilization of externally fertilizing species, such as marine invertebrates, fish, and frogs. Recent studies demonstrate that the requirement for Src-family kinases in activation of the mammalian egg is different from lower species, and the objective of this study was to test the role of the Fyn kinase in the mouse egg activated by intracytoplasmic sperm injection (ICSI). An Src homology 2 (SH2) domain containing fusion protein was used to suppress Fyn function in the mouse zygote following ICSI. Eggs injected with the Fyn SH2 domain at an intracellular concentration of 4-8 $\mu \mathrm{M}$ exhibited reduced developmental potential with $100 \%$ of the zygotes being arrested following the first or the second cleavage. At higher concentrations, the protein blocked pronuclear congression and the zygotes remained at the pronuclear stage. The $\mathrm{SH} 2$ domain had no effect on sperm-induced calcium oscillations in distinct contrast to its effect on the eggs of lower species. The results indicate that the $\mathrm{SH} 2$ domain of Fyn kinase plays an important role in pronuclear congression as well as early cleavage events and that this effect appears not to involve disruption of calcium oscillations.

Reproduction (2006) 132 413-421
\end{abstract}

\section{Introduction}

Egg activation involves a series of preprogrammed biochemical events that function to establish a block to polyspermy, incorporate the sperm into the ooplasm, release the oocyte from $\mathrm{MII}$ arrest, and then initiate the mitotic cell cycle. In addition, pathways are triggered that are critical to activation of the zygotic genome and later the developmental events. Signal transduction cascades involving Src-family protein tyrosine kinases (PTKs), such as Fyn, Src, and Yes have been shown to play a major role during egg activation and early development in species that fertilize externally, such as marine invertebrates, amphibians, and fish (Sato et al. 2000a, Wu \& Kinsey 2001, Runft et al. 2002). Src-family PTKs are cytoplasmic enzymes that can be targeted to plasma membrane microdomains where they typically act to transduce signals from external stimuli (Bromann et al. 2004). In non-mammalian species, Fyn and/or Src kinase are activated rapidly after fertilization and function in triggering the sperm-induced calcium transient that initiates the egg activation process (Carroll et al. 1999, Giusti et al. 1999, 2000, Sato et al. 2000b). Later stages of egg activation such as pronuclear fusion and mitosis also require PTK activity, although the specific kinases involved in these steps have not been identified (Moore \& Kinsey 1995, Wright \& Schatten 1995). Once development has begun, Fyn and Yes are required for cell movements involved in epiboly (Sharma et al. 2005), while Src and Yes function during cell intercalation and blastopore closure (Denoyelle et al. 2001).

The role of Src-family PTKs in mammalian fertilization has received less study than in non-mammals, but it is clear that the role of these kinases during mammalian egg activation is different. For example, while mammalian eggs express Fyn, Yes, and in some cases, Src (Talmor et al. 1998, Talmor-Cohen et al. 2004a), the results of two different studies indicate that these kinases are not required for the unique sperm-induced calcium oscillations (Kurokawa et al. 2004a, 2004b, Mehlmann \& Jaffe 2005), which trigger egg activation in mammals (Carroll 2001). Instead, these calcium oscillations are triggered directly by a sperm-borne phospholipase that does not require PTK regulation (Cox et al. 2002). The role of Src-family PTKs in later stages of mammalian fertilization has been addressed primarily using parthenogenetic activation. Studies in mouse and rat demonstrate that agents, which suppress Src-family kinase activation, also inhibit the MII/anaphase transition induced by parthenogenetic activation in vitro. 
In addition, microinjection of active Fyn kinase has been shown to stimulate meiosis resumption in mouse and rat (Sette et al. 2002, Talmor-Cohen et al. 2004b). A second requirement for Src-family PTK activity at S- or S/G2phase of the first mitotic division has been demonstrated using chemical inhibitors, such as genistein (Besterman \& Schultz 1990, Jacquet et al. 1995). Together, these observations indicate that Src-family kinases, such as Fyn may play an important role in development of the mammalian zygote, but it is unclear whether all these pathways are activated in response to the sperm.

The Src homology $2(\mathrm{SH} 2)$ domain is a P-Tyr-binding region found in many proteins. Src-family PTKs have a single copy of this domain adjacent to the catalytic domain where it functions in maintaining the configuration of the kinase in either the active or the inactive state (Bradshaw \& Waksman 1993, Zheng et al. 2000, 2002, Wong et al. 2004). This domain also functions in protein-protein interactions with other signaling molecules, such as cell-surface receptors and downstream effectors. These features allow microinjected $\mathrm{SH} 2$ domain-containing fusion proteins to suppress activation of the kinase in vivo (Superti-Furga \& Courtneidge 1995, Kinsey et al. 2003) or block its interaction with downstream effectors to exert a dominant-negative effect (Chuang et al. 1994, Roche et al. 1995). This approach has been used to demonstrate a role for $\mathrm{SH} 2$ domain function in Pl1-3 kinase activation leading to progesterone-induced MI resumption (Muslin et al. 1993, Katzav et al. 1995), as well as the function of Fyn and/or Src in egg activation in lower species.

The objective of the present study was to determine whether the Fyn and Src kinases play an important role in mammalian egg activation by sperm. The present study was performed with eggs fertilized by intracytoplasmic sperm injection (ICSI), since this procedure allowed timed activation, which facilitated statistical analysis of many aspects of the zygotic development. The approach was to use $\mathrm{SH} 2$ domain-containing fusion proteins as dominant-negative inhibitors of PTK function and monitor the effect on egg activation and development to the blastocyst stage. The results indicate that Fyn kinase plays an important role in events required for pronuclear congression and initiation of cell division.

\section{Materials and Methods}

B6D2F1 and C57BL/6 mice were housed in a temperature- and light-controlled room on a $14 \mathrm{~h}$ light: $10 \mathrm{~h}$ darkness cycle and experiments were conducted in accordance with the Guide for the Care and Use of Laboratory Animals published by the National Academy of Sciences. Females (6-8 weeks old) were induced to superovulate by i.p. injection of $5 \mathrm{IU}$ pregnant mare serum gonadotropin, followed by $5 \mathrm{IU}$ human chorionic gonadotropin (hCG), $48 \mathrm{~h}$ later, both of which were obtained from Sigma. Mature oocytes were collected at
14-16 host-hCG administration by the dissection of oviducts. Cumulus cells were removed by brief exposure to serum-free modified human tubal fluid (mHTF) medium (Irvine Scientific, Santa Ana, CA, USA) containing $30 \mathrm{IU} / \mathrm{ml}$ hyaluronidase (Sigma) and maintained in P-1 medium (Irvine Scientific, Santa Ana, CA, USA) supplemented with $10 \%$ fetal bovine serum (Atlanta Biologicals, Lawrenceville, GA, USA).

For in vitro fertilization (IVF), cauda epididymal sperm from C57BL/6 mice were capacitated by incubation in modified Tyrode's medium (Summers et al. 1995) equilibrated with $5 \% \mathrm{CO}_{2}$ at $37{ }^{\circ} \mathrm{C}$ for $90 \mathrm{~min}$. Capacitated sperm were collected from the top of the incubation tube and added to dishes $\left(1 \times 10^{6} \mathrm{sperm} / \mathrm{ml}\right)$ containing MII oocytes in $\mathrm{KSOM}^{\mathrm{AA}}$ (Chemicon International, Temecula, CA, USA) with $10 \%$ fetal calf serum. After incubation for $90 \mathrm{~min}$, the fertilized eggs were removed and cultured in droplets of fresh medium.

For ICSI, cauda epididymal sperm of male C57BL/6 mice were cryopreserved using 3\% skim milk and $18 \%$ raffinose (Sigma) in water as described earlier (Nakagata 1992). Samples were thawed in a $37^{\circ} \mathrm{C}$ water bath for $2 \mathrm{~min}$, centrifuged at $15000 \mathrm{~g}$ for $5 \mathrm{~min}$, resuspended, and overlain with mHTF medium to allow active sperm to swim up. Sperm were suspended in mHTF containing $6 \%$ polyvinyl pyrrolidone (MW 360; Sigma). A single sperm was aspirated into an injection pipette of $\sim 7 \mu \mathrm{m}$ inner diameter and positioned such that its neck was at the opening of the pipette. The head was removed by applying a piezo pulse so that the head separated from the tail. A group of sperm heads were pooled into a droplet of injection buffer to which was added various test reagents. Single sperm heads were then aspirated into a mercury-filled pipette for injection into MII oocytes. ISCl was then performed with a piezo impact micromanipulator (Prime Tech Ltd, Ibaraki-ken Japan). The zygotes with injected sperm head were incubated in droplets of $\mathrm{KSOM}^{\mathrm{AA}}$ medium with $10 \%$ fetal calf serum, which was overlain with mineral oil and equilibrated with humidified air $/ 5 \% \mathrm{CO}_{2}$ at $37{ }^{\circ} \mathrm{C}$. The results of several experiments demonstrated that ICSI (sperm head only) resulted in high rates $(92.0 \%)$ of egg activation as defined by the presence of two fully developed pronuclei and that $41.6 \%$ developed to the blastocyst stage. Injection volumes were measured at the end of each experiment by photographing the pipette before and after injection, and then calculating the volume delivered by the distance the mercury meniscus had changed. Injection volumes were 1.3-1.5 pl.

For immunofluorescence, eggs were fixed in a mixture of $2 \%$ paraformaldehyde $+0.5 \%$ picric acid (Zamboni's fixative; Andersen et al. 1988) in PBS for $1 \mathrm{~h}$, rinsed three times with PBS containing $0.1 \%$ Triton X-100, and permeabilized for $0.5 \mathrm{~h}$ with PBS containing $0.5 \%$ Triton X-100. The eggs were then blocked for $1 \mathrm{~h}$ with PBS containing $0.1 \%$ Triton $X-100,3 \mathrm{mg} / \mathrm{ml}$ BSA, and $0.15 \mathrm{M}$ glycine. Fyn kinase was detected with a rabbit 
polyclonal antibody directed against the $\mathrm{N}$-terminal domain of the protein (anti-Fyn-3; Santa Cruz Biotechnology, Inc., Santa Cruz, CA, USA) at a concentration of $2.0 \mu \mathrm{g} / \mathrm{ml}$ during overnight incubation. After washing, bound antibody was detected with FITC-conjugated anti-rabbit IgG (Santa Cruz Biotechnology). Controls were incubated with the secondary antibody only. Fluorescence was detected with a Zeiss LSM 510 onfocal microscope using a $488 \mathrm{~nm}$ laser.

In order to detect developmental changes in $\left[\mathrm{Ca}^{2+}\right]_{i,}$ eggs were injected with a sperm head mixed with calcium green-dextran to achieve intracellular concentrations of approximately $0.5 \mu \mathrm{M}$. The eggs were then monitored by confocal fluorescence microscopy on a Nikon TE2000U microscope using a $488 \mathrm{~nm}$ Spectra Physics (Mountainview, CA, USA) laser. Time-course measurements were made with a $20 \times$ super fluor objective with pinhole settings set to obtain a theoretical $24 \mu \mathrm{m}$ optical section through the equator of the embryo. Emitted fluorescence was recorded at 15-s intervals and separate images were collected using transmitted light, to obtain a DIC image, and a $515 / 30 \mathrm{~nm}$ band pass filter to obtain calcium green fluorescence. The fluorescence intensity of the zygote was quantitated from digital images by centering an elliptical measurement area over the entire zygote, and integrated pixel density was calculated by Metamorph 6.2 (Universal Imaging Corp., Downington, PA, USA).

A GST fusion protein encoding the $\mathrm{SH} 2$ domain of Fyn was prepared as described earlier (Kinsey \& Shen 2000). The GST-SH2 domain of Src was a generous gift from S. Courtneidge (Sugen, Inc., San Francisco, CA, USA). The fusion proteins were expressed in bacteria $(\mathrm{DH} 5 \alpha)$, purified by affinity chromatography on a glutathioneagarose (Sigma) column, concentrated and dialyzed into injection buffer consisting of $0.15 \mathrm{M} \mathrm{KCl}, 3 \mathrm{mM} \mathrm{NaCl}$ $10 \mathrm{mM} \mathrm{KH}_{2} \mathrm{PO}_{4}(\mathrm{pH} 7.2), 1 \mathrm{mM}$ glutathione, and $80 \mathrm{mM}$ sucrose. Protein content was determined by the BCA (bicinchoninic acid) assay (Pierce Biotechnology Inc, Rockford, IL, USA).

The PTK inhibitors PP2 (4-amino-5(4-chlorophenyl)-7(t-butyl)pyrazolo[3,4-d] pyrimidine), SU6656 (2-oxo3(4,5,6,7-tetrahydro- $1 H$-indol-2-ylmethylene)-2,3-dihydro- $1 \mathrm{H}$-indole5-sulfonic acid dimethylamide), and the inactive homolog PP3 (4-amino-7-phenylpyrazol[3,4d] pyrimidine) were obtained from Calbiochem (La Jolla, CA, USA) and solubilized in DMSO.

\section{Results}

\section{Expression of Fyn kinase in the mouse oocyte}

Src-family kinases are expressed at different levels in a wide variety of cells as cytoplasmic or membraneassociated proteins of $59-62 \mathrm{kDa}$. The members of this family can be reliably detected by antibodies directed against the $\mathrm{N}$-terminal unique domain, which distinguishes each member of this closely related family of kinases. The anti-Fyn-3 antibody is directed against aa 28-48, a sequence unique to Fyn, and this antibody was used to detect the $59 \mathrm{kDa}$ protein in a sample of cumulusfree MII oocytes (Fig. 1, lane A). Antibody binding was blocked by the presence of excess peptide antigen (lane B), confirming that the binding was specific.

The anti-Fyn-3 antibody was also used to detect the Fyn protein by confocal immunofluorescence of MII oocytes and zygotes at different stages of development (Fig. 2). Fyn was enriched in the cortex of the MII oocyte with the cytoplasm exhibiting less intense fluorescence, which was uniform in character (Fig. 2A). In general, the distribution of Fyn in the mouse egg resembled with that previously reported for the rat egg (Talmor et al. 1998) except that specific localization of Fyn to the meiotic spindle was not observed in the mouse oocyte under these conditions. After fertilization, Fyn remained concentrated in the egg cortex, including the region of sperm incorporation (Fig. 2B, arrow), as well as in the region of the MII spindle. The sperm also contain Fyn kinase and this was evident in the midpiece of the fertilizing sperm. Once MII was completed, the distribution of Fyn in the cytoplasm became less uniform, with condensed regions exhibiting higher staining intensity (Fig. 2C). Fyn remained concentrated in the zygote cortex with some accumulation just deep to the polar bodies, which themselves were intensely stained. At the late pronuclear stage, the regions of condensed cytoplasmic fluorescence became more abundant (Fig. 2D) and Fyn accumulation was frequently associated with the pronuclear envelope (arrow) and the nucleoli. After the first mitosis, Fyn remained concentrated in the cortex of each blastomere, including the

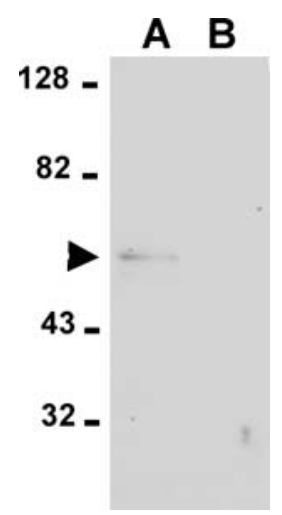

Figure 1 Detection of Fyn kinase in the mouse oocyte. Western blot analysis was performed on samples of MII oocytes resolved on a $10 \%$ SDS gel and blotted to an Immobilon-P membrane. One lane (50 oocytes) was incubated with the anti-Fyn-3 antibody at $1 \mu \mathrm{g} / \mathrm{ml}$ (A) while the other lane (50 oocytes) was incubated with the anti-Fyn-3 antibody $+1 \mathrm{mM}$ synthetic peptide antigen (RYGSDPTPQHYPSFGVT) as a control (B). Bound antibody was detected with goat anti-rabbit IgG coupled to horseradish peroxidase followed by chemiluminescence detection. The position of the $59 \mathrm{kDa}$ band is indicated by the arrow. 

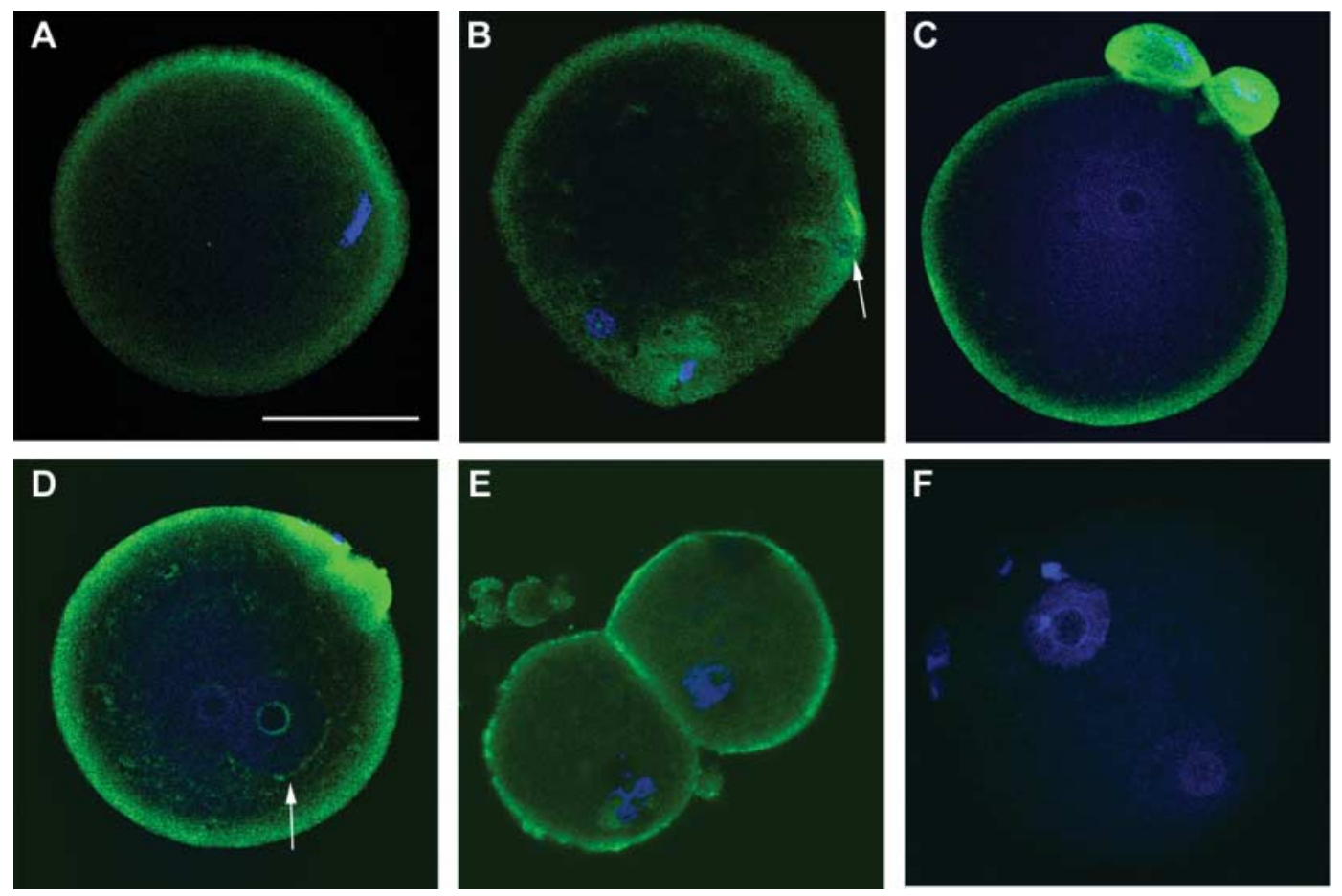

Figure 2 Detection of Fyn kinase by confocal immunofluorescence microscopy. MII oocytes were fixed before (A), and at different times after in vitro fertilization; $2 \mathrm{~h}(\mathrm{~B}), 6 \mathrm{~h}(\mathrm{C}), 12 \mathrm{~h}(\mathrm{D}, \mathrm{F}), 24 \mathrm{~h}(\mathrm{E})$, permeabilized, and blocked as described in 'Materials and Methods', then incubated with the antiFyn-3 antibody followed by FITC-conjugated goat anti-rabbit IgG (A-E), or with secondary antibody only (F). The position of the meiotic chromosomes and pronuclei was detected by DAPI staining (blue). Magnification is indicated by the bar, which represents $50 \mu \mathrm{m}$. The position of the fertilizing sperm is indicated in (B) by the white arrow. The arrow in (D) indicates a region of condensed Fyn-immunofluorescence typical of the late pronuclear stage.

newly formed border between blastomeres. The central cytoplasm of the two-cell stage embryo contained Fyn that was evenly distributed with very few of the regions of condensed immunofluorescence, typical of the pronuclear stages (Fig. 2E).

\section{Role of Src-family kinases during zygotic development}

Inhibitor studies performed in oocytes from mouse and rat have implicated Src-family PTKs in events required for MII resumption following parthenogenetic activation (Sette et al. 2002, Talmor-Cohen et al. 2004a). After fertilization, PTK inhibitors have been shown to block zygote development during or just prior to DNA synthesis resulting in an arrest of development prior to the two-cell stage (Besterman \& Schultz 1990, Jacquet et al. 1995). Since the performance of those studies, a new generation of PTK inhibitors with higher specificity for the Src-family PTKs has been developed and, in an effort to more carefully define the role of Src-family PTKs in these processes, we tested the effect of two chemical inhibitors that are very specific for the Src-family kinases, PP2, and SU6566, as well as a less active analog, PP3, on the development of eggs fertilized by the sperm. Despite testing a wide range of concentrations, we were unable to find a concentration of these inhibitors that affected the development without causing a diverse set of defects leading to abnormal nuclear morphology and cytoplasmic blebbing (not shown). The observed limitations of these compounds with regard to toxicity as well as the possibility that they affect other classes of kinases prompted us to perform further studies using reagents like domain-specific fusion proteins, which are more specific.

\section{Role of Src and Fyn kinases during early development}

While the above experiment with chemical inhibitors provided some indication that Src-family PTKs may play a role in the development of activated zygote, a more specific test of the role of Fyn and Src kinases was performed with GST fusion proteins encoding the $\mathrm{SH} 2$ domain of these kinases as dominant-negative inhibitors of kinase function in vivo. These reagents have been extensively used in the study of kinase function via microinjection into unfertilized eggs at concentrations ranging from 2 to $20 \mu \mathrm{M}$ (Giusti et al. 1999, 2003, Runft et al. 1999, Kinsey \& Shen 2000). In order to more clearly test the role of these kinases in MII resumption and obtain a more synchronized population of zygotes, this series of experiments utilized ICSI to fertilize the eggs. In order to maintain high rates of development, it 
was important to minimize the number of times the egg was penetrated with a pipette, hence, the fusion protein was dialyzed into injection buffer, and sperm heads added to a droplet of this mixture. Individual sperm heads were then recovered and injected into the oocytes. Injection volumes were approximately $0.65 \%$ of the egg volume, which would result in a final intracellular concentration of fusion protein between 4 and $16 \mu \mathrm{M}$. The zygotes were subsequently cultured and examined periodically for developmental progress. The results presented in Table 1 demonstrate that the $\mathrm{SH} 2$ domain fusion proteins did not interfere with the early stages of egg activation triggered by ICSI, such as meiosis resumption and pronucleus formation. However, later steps during egg activation were affected by the GST-Fyn-SH2 protein, which suppressed mitosis in a concentration-dependent manner and blocked blastocyst formation at all concentrations tested. The GST-Src-SH2 fusion protein had no significant effect on activation or on subsequent development; however, the combination of GST-Fyn-SH2 and GST-Src-SH2 inhibited cleavage and development at a level similar to that of GST-Fyn-SH2 alone.

Examination of the embryos following GST-Fyn-SH2 injection at the lowest concentration revealed that the embryos were mostly arrested between the two- and four-cell stage and none reached the compaction stage (Fig. 3b). At higher concentrations (8 and $16 \mu \mathrm{M})$, zygotes formed normal-appearing pronuclei by $6-\mathrm{h}$ post-ICSI, and remained at that stage for as long as $48 \mathrm{~h}$ before degenerating (Fig. $3 \mathrm{c}$ ). During this period, the male and the female pronuclei remained adjacent to each other but there was no sign of nuclear envelope breakdown. The GST-Fyn-SH2 protein did not appear to prevent the male and the female pronuclei from migrating into proximity with each other, but further nuclear events leading to nuclear envelope breakdown, syngamy, and mitosis were prevented.
Previous studies in the zebrafish system have demonstrated that injection of the GST-Fyn-SH2 fusion protein suppressed the activation of Src-family PTKs following fertilization (Kinsey et al. 2003). In order to determine whether this fusion protein might also have effects on the subcellular distribution of the endogenous Fyn kinase, we performed immunolocalization of Fyn in zygotes fixed following injection of GST-Fyn-SH2 or GST as a control. As seen in Fig. 4, the distribution of Fyn in zygotes arrested following GST-Fyn-SH2 injection was similar to control embryos in that the majority of immunofluorescence was associated with the egg cortex. Both control and GST-Fyn-SH2-injected zygotes exhibited significantly less evidence of cytoplasmic condensations of Fyn immunofluorescence such as those evident in Fig. 2D. This highlights the fact that microinjection of proteins is with consequence in the mammalian zygote, but the specific, developmentally relevant effects of GST-Fyn-SH2 probably do not involve perturbations in the subcellular distribution of Fyn protein.

\section{Role of Fyn activity on calcium signaling following ICSI}

The observed effects of Fyn $\mathrm{SH} 2$ domain-containing fusion proteins on egg activation suggested that the Fyn protein kinase plays an important role in signal transduction after fertilization. Since numerous studies have implicated Fyn during calcium signaling in eggs of invertebrates and lower vertebrates (Runft et al. 2002), and calcium signaling is also critical for activation of the mammalian egg (Jones \& Nixon 2000, Ducibella et al. 2002, Carroll et al. 2004), it was important to test whether GST-Fyn-SH2 interfered with calcium signaling following ICSI in the mouse system. In this experiment, the fluorescent calcium reporter calcium green-dextran was added to the GST

Table 1 Effects of the SH2 domain from Src and Fyn on mouse oocyte activation and embryo development.

\begin{tabular}{|c|c|c|c|c|c|}
\hline Injected & $n$ & Activated (\%) & 2-cell (\%) & 3-4- cell (\%) & Blastocyst (\%) \\
\hline GST, $16 \mu \mathrm{M}$ & 63 & 92 & 78 & 74 & 41 \\
\hline \multicolumn{6}{|l|}{ GST-Fyn-SH2 } \\
\hline $4 \mu \mathrm{M}$ & 20 & 100 & 70 & $25^{*}$ & $0 *$ \\
\hline $8 \mu \mathrm{M}$ & 20 & 90 & $40^{*}$ & $14^{*}$ & $0^{*}$ \\
\hline $16 \mu \mathrm{M}$ & 42 & 100 & $0 *$ & $0^{*}$ & $0^{*}$ \\
\hline \multicolumn{6}{|l|}{ GST-Src-SH2 } \\
\hline $4 \mu \mathrm{M}$ & 34 & 97 & $97 *$ & 91 & 56 \\
\hline $16 \mu \mathrm{M}$ & 27 & 93 & 89 & 88 & 56 \\
\hline \multicolumn{6}{|c|}{ GST-Fyn-SH2 + GST-Src-SH2 } \\
\hline $4 \mu \mathrm{M}$ & 20 & 100 & 69 & $25^{*}$ & $0^{*}$ \\
\hline $8 \mu \mathrm{M}$ & 23 & 92 & $5 *$ & $13 *$ & $0^{*}$ \\
\hline $20 \mu \mathrm{m}$ & 29 & 100 & $9 *$ & $0^{*}$ & $0^{*}$ \\
\hline
\end{tabular}

GST fusion proteins encoding the SH2 domain of Fyn or Src were injected together with a sperm head into MII oocytes. Zygotes were examined after 30 -min culture to assess viability and eggs, which were obviously damaged by the ICSI procedure were removed. Viable zygotes $(n)$ were then cultured for 5-6 days. Zygotes were judged to have been activated if the second polar body was extruded and if two expanded pronuclei were visible at $6 \mathrm{~h}$ after ICSI. Progression through the two-cell stage was determined at $30 \mathrm{~h}$ after ICSI. Progression through the three- to four-cell stage was determined at 54-h post-ICSI, and embryos were scored for progression to the blastocyst stage at 5-6 days post-ICSI. $\left(^{*}\right)$ indicates that the value is significantly different from control as determined by ANOVA $(P<0.05)$. 
A

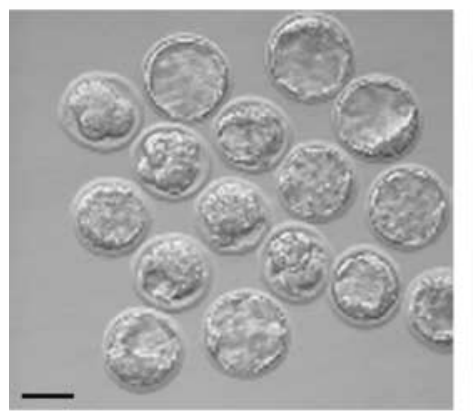

B

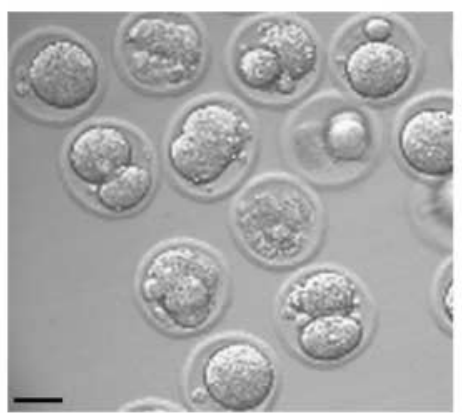

C

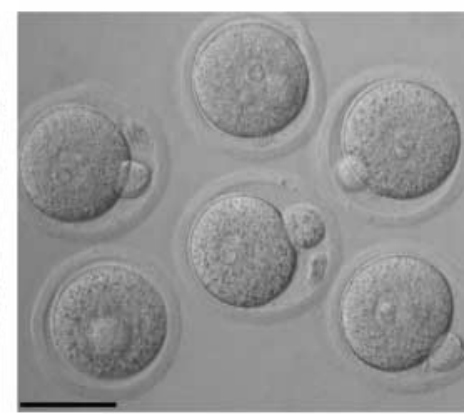

Figure 3 Effect of GST-Fyn-SH2 on development following ICSI. MII oocytes were injected with injection buffer containing a sperm head and either GST at an intracellular concentration of $16 \mu \mathrm{M}$ (A) or GST-Fyn-SH2 at $4 \mu \mathrm{M}$ (B) or $16 \mu \mathrm{M}(\mathrm{C})$, then cultured as described in 'Materials and Methods'. By 5-6 days post-ICSI, the control embryos were entering the blastocyst stage, while zygotes injected with GST-Fyn-SH2 were arrested at the one- to fourcell stage (B). Zygotes injected with GST-Fyn-SH2 at higher concentration $(16 \mu \mathrm{M})$ completed meiosis as indicated by the presence of a second polar body and two pronuclei, but did not undergo cleavage during $48 \mathrm{~h}$ of culture (C). Magnification is indicated by the bar, which represents $50 \mu \mathrm{m}$.

(control) or GST-Fyn-SH2 fusion protein solutions at a concentration of $25 \mu \mathrm{M}$. Once groups of 5-10 MII oocytes were injected with the mixture of sperm head, fusion protein, and calcium green-dextran, the zygotes were monitored by confocal fluorescence microscopy with scans recorded at 15-s intervals. The changes in fluorescence intensity of each zygote were measured throughout the time-series of images by quantitating integrated pixel intensity with Metamorph 6.2 software. The results of typical recordings are shown graphically in Fig. 5. It is clear that normal calcium oscillations occurred in zygotes containing both control (GST) and experimental (GST-Fyn-SH2) proteins. In order to compare the pattern of calcium oscillations between these groups, we quantitated the number of calcium transients during the first $2 \mathrm{~h}$ after sperm injection. The average number of transients in the control group $(n=20)$ was 7.5 , while the average in the GST-Fyn$\mathrm{SH} 2$ group $(n=49)$ was 8 . This difference was not significant as determined by Mann-Whitney rank sum test $(P=0.561)$. These results are consistent with the recently published experiments testing these fusion proteins in eggs activated by normal fertilization (Kurokawa et al. 2004a, 2004b, Mehlmann \& Jaffe 2005) and indicate that Fyn kinase is not required for normal calcium oscillations in eggs activated by fertilization or ICSI. The results indicate that the effects of GST-Fyn-SH2 on pronuclear congression and cleavage do not likely involve suppression of calcium signaling.

\section{Discussion}

Numerous studies involving chemical inhibitors, dominant-negative fusion proteins, and microinjection of exogenous PTKs have demonstrated that Src-family PTKs, including Fyn and possibly others, play a critical role in the activation of eggs from non-mammalian species. Examples include a demonstrated role for PTKs in events such as sperm-egg fusion (Sakakibara et al. 2005), the sperm-induced calcium transient (Abassi et al. 2000), pronuclear migration and fusion (Moore \& Kinsey 1995, Wright \& Schatten 1995), as well as developmental competence (Livingston et al. 1998, Sharma et al. 2005). The extent to which these pathways are active in mammalian fertilization is now the subject of much investigation. Analysis of mammalian egg activation using chemical inhibitors (PP2 and SU6656), which are reasonably specific for Src-family PTKs (Bain et al. 2003) has demonstrated that MII resumption induced parthenogenetically by calcium ionophore or by Tr-kit required Src-family PTK activity (Sette et al. 2002, Talmor-Cohen et al. 2004b). Once meiosis was completed, suppression of PTKs in the mouse zygote by genistein caused a second block in which DNA synthesis was suppressed and the zygotes failed to exit S-phase of the cell cycle (Besterman \& Schultz 1990).

In an effort to rule out the possible non-specific effects of chemical inhibitors and to more precisely identify the
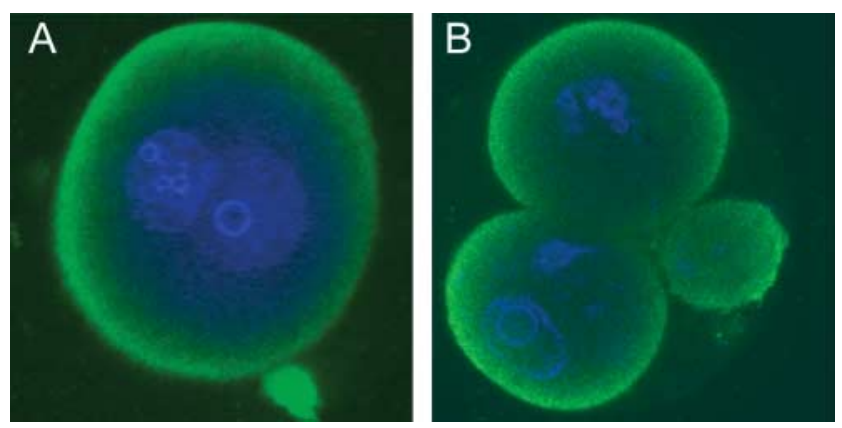

Figure 4 Effect of injected GST-Fyn-SH2 on the distribution of Fyn kinase in the zygote. Zygotes injected with a sperm head together with GST-Fyn-SH2 (A) or GST (B) at an intracellular concentration of $16 \mu \mathrm{M}$ were fixed at 30-h post-ICSI and immunolabeled with the Fyn-3 antibody followed by goat anti-rabbit IgG-alexa 488, which localized the native Fyn protein by green fluorescence. Nuclear structure were localized with DAPI and appear blue. 

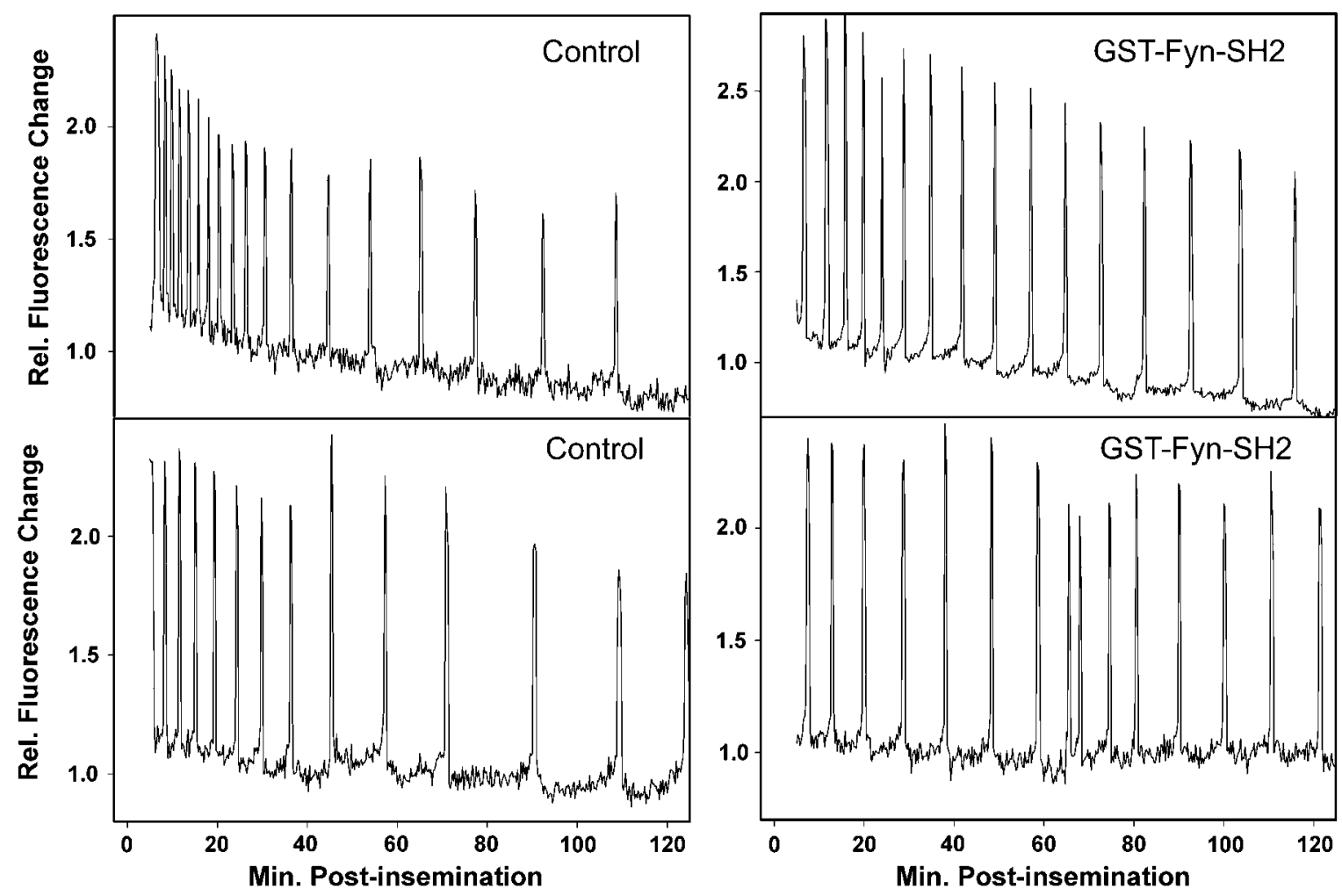

Figure 5 Effect of GST-Fyn-SH2 on calcium signaling following ICSI. MII oocytes were injected with a sperm head in buffer containing calcium greendextran and either GST (control) or GST-Fyn-SH2 at $16 \mu \mathrm{M}$. Images of calcium-induced fluorescence were recorded at 15-s intervals by confocal fluorescence microscopy. Fluorescence was quantitated by measuring average pixel intensity over each individual oocyte using Metamorph 6.2 software. Panels represent fluorescence data from two typical zygotes from the control (left) and GST-Fyn-SH2 (right)-injected groups.

kinases involved in these events, we made use of the GST fusion proteins encoding the $\mathrm{SH} 2$ domain of Fyn and Src, which were microinjected into the egg with the fertilizing sperm head. These fusion protein reagents are less likely than chemical inhibitors to cause non-specific effects and they also allow some discrimination between a requirement for Fyn and Src (Bradshaw \& Waksman 1993). The use of ICSI, instead of IVF, avoided possible complications regarding sperm motility or sperm-egg fusion. The results demonstrated that injection of the $\mathrm{SH} 2$ domain of Fyn or Src kinase into mouse oocytes during ISCI had no effect on MII resumption, and normal-appearing male and female pronuclei were formed. This result differed from published reports described above that used chemical inhibitors to block MII resumption following parthenogenetic activation. The most likely explanation for this discrepancy is that the fertilizing sperm might activate multiple signaling pathways more completely than parthenogenetic treatments and therefore suppression of Src-family PTKs could not block the activation of eggs fertilized by sperm. A second possibility is that MII resumption requires the catalytic activity of Src-family PTKs, but that their role does not require the $\mathrm{SH} 2$ domain.

While the Fyn $\mathrm{SH} 2$ domain did not block meiosis resumption or the formation of pronuclei, this fusion protein greatly affected the developmental potential. The lower range of concentrations of GST-Fyn-SH2 caused most zygotes to be arrested during the first two cleavages and none reached the blastocyst stage. At higher concentrations, zygotes were arrested at the pronuclear stage with both male and female pronuclei remaining adjacent in the center of the zygote. These results resemble findings made in marine invertebrate eggs where chemical PTK inhibitors as well as GST-Fyn-SH2 caused a failure of pronuclei to fuse (Moore \& Kinsey 1995, Kinsey \& Shen 2000). However, in the invertebrate system, the male pronuclei were also unable to migrate to the vicinity of the female pronucleus, while the present results in mouse indicated that pronuclear migration was adequate to position both pronuclei in the center of the zygote. The defect induced by Fyn-SH2 protein also resembled the phenotype recently described to result from knockout of the Zar-1 gene (Wu et al. 2003) and the fue gene in zebrafish (Dekens et al. 2003), raising the possibility that Src-family PTKs may function in the pathways controlled by these genes. In any case, the effect of Fyn-SH2 protein appeared not to involve effects on calcium signaling, since the pattern of calcium oscillations was not altered significantly by the injection of this protein. We also investigated the possibility 
that the injected $\mathrm{SH} 2$ domain could cause the redistribution of the endogenous Fyn kinase from the egg cortex. However, immunofluorescence analysis did not reveal any significant difference in the pattern of between GST-Fyn-SH2 and control (GST)-injected zygotes. It seems more likely that the effect induced by the GST-Fyn-SH2 protein was a result of its ability to suppress activation of the Fyn kinase as described in the zebrafish egg (Kinsey et al. 2003).

In summary, in the mammalian zygote, the primary requirement for Src-family PTK activity, as determined by interruption of the function of the $\mathrm{SH} 2$ domain, relates to the formation of the zygote nucleus and progression through the cell cycle. There is precedent for a role of Src-family PTKs in cell division of somatic cells since inhibitor studies have established that SFK activity is required at the G1/S transition (Bromann et al. 2004) and, in some cases, at G2/M. Future studies will hopefully establish the exact mechanism by which the kinases function in development of the zygote.

\section{Acknowledgements}

This work was supported by NIH-CHD 14846 and Conrad Twinning Grant MFG-03-66. We would like to acknowledge Elinor Macgreggor and Lynda McGinnis for assistance with the confocal microscopy. The authors declare that there is no conflict of interest that would prejudice the impartiality of this scientific work.

\section{References}

Abassi Y, Carroll D, Giusti AF, Belton R \& Foltz KR 2000 Evidence that Src-type tyrosine kinase activity is necessary for calcium release at fertilization in sea urchin eggs. Developmental Biology 15 206-219.

Andersen J, Orntoft TF \& Poulsen HS 1988 Immunohistochemical demonstration of estrogen receptors (ER) in formalin fixed, paraffinembedded human breast cancer tissue by use of a monoclonal antibody to ER. Journal of Histochemistry and Cytochemistry 12 1553-1560.

Bain J, Mclauchlan H, Elliott M \& Cohen P 2003 The specificities of protein kinase inhibitors: an update. Biochemical Journal 371 199-204.

Besterman B \& Schultz RM 1990 Regulation of mouse preimplantation development: effect of genistein, an inhibitor of tyrosine protein phosphorylation on cleavage of one-cell embryos. Journal of Experimental Zoology 256 44-53.

Bradshaw JM \& Waksman G 1993 Molecular recognition by $\mathrm{SH} 2$ domains. Advances in Protein Chemistry 61 161-210.

Bromann PA, Korkaya H \& Courtneidge SA 2004 The interplay between Src family kinases and receptor tyrosine kinases. Oncogene 23 7957-7968.

Carroll J 2001 The initiation and regulation of $\mathrm{Ca}^{2+}$ signalling at fertilization in mammals. Seminars in Cell \& Developmental Biology 12 37-43.

Carroll DJ, Albay DT, Terasaki M, Jaffe LA \& Foltz KR 1999 Identification of PLCgamma-dependent and -independent events during fertilization of sea urchin eggs. Developmental Biology 206 232-247.
Carroll J, FitzHarris G, Marangos P \& Halet G $2004 \mathrm{Ca}^{2+}$ signalling and cortical re-organisation during the transition from meiosis to mitosis in mammalian oocytes. European Journal of Obstetrics, Gynecology, and Reproductive Biology 115 S61-S67.

Cox LJ, Larman MG, Saunders CM, Hashimoto K, Swann K \& Lai FA 2002 Sperm phospholipase Czeta from humans and cynomoigus monkeys triggers $\mathrm{Ca}^{2+}$ oscillations, activation and development of mouse oocytes. Reproduction 124 611-623.

Chuang LM, Hausdorff SF, Myers MG, White MF, Birnbaum MJ \& Kahn CR 1994 Interactive roles of Ras, insulin receptor substrate-1, and proteins with Src homology-2 domains in insulin signaling in Xenopus oocytes. Journal of Biological Chemistry 269 27645-27649.

Dekens MP, Pelegri FJ, Maischein HM \& Nusslein-Volhard C 2003 The maternal-effect gene futile cycle is essential for pronuclear congression and mitotic spindle assembly in the zebrafish zygote. Development 130 3907-3916.

Denoyelle M, Valles A, Lentz D, Thiery JP \& Boyer B 2001 Mesodermindependent regulation of gastrulation movements by Src tyrosine kinase. Differentiation 69 38-48.

Ducibella T, Huneau D, Angelichio E, Xu Z, Schultz RM, Kopf GS, Fissore R, Madoux S \& Ozil JP 2002 Egg-to-embryo transition is driven by differential responses to $\mathrm{Ca}^{2+}$ oscillation number. Developmental Biology 250 280-291.

Giusti AF, Carroll DJ, Abassi YA, Terasaki M, Foltz KR \& Jaffe LA 1999 Requirement of a Src family kinase for initiating calcium release at fertilization in starfish eggs. Journal of Biological Chemistry 274 29318-29322.

Giusti AF, Xu WQ, Hinkle B, Terasaki M \& Jaffe LA 2000 Evidence that fertilization activates starfish eggs by sequential activation of a Srclike kinase and phospholipase Cgamma. Journal of Biological Chemistry 275 16788-16794.

Giusti AF, O'Neill FJ, Yamasu K, Foltz KR \& Jaffe LA 2003 Function of a sea urchin egg Src family kinase in initiating $\mathrm{Ca}^{2+}$ release at fertilization. Developmental Biology 256 367-378.

Jacquet P, Saint-Georges L, Barrio S \& Baugnet-Mahieu L 1995 Morphological effects of caffeine, okadaic acid, and genistein in one cell mouse embryos blocked in G2 by X-irradiation. International Journal of Radiation Biology 67 347-358.

Jones KT \& Nixon VL 2000 Sperm-induced $\mathrm{Ca}^{2+}$ oscillations in mouse oocytes and eggs can be mimicked by photolysis of caged inositol 1,4,5-trisphosphate: evidence to support a continuous low level production of inositol 1,4,5-trisphosphate during mammalian fertilization. Developmental Biology 225 1-12.

Katzav S, Packham G, Sutherland M, Aroca P, Santos E \& Cleveland JL 1995 Vav and Ras induce fibroblast transformation by overlapping signaling pathways which require c-Myc function. Oncogene 11 1079-1088.

Kinsey WH \& Shen SS 2000 Role of the Fyn kinase in calcium release during fertilization of the sea urchin egg. Developmental Biology 225 253-264.

Kinsey WH, Wu W \& Macgregor E 2003 Activation of Src-family PTK activity at fertilization: role of the $\mathrm{SH} 2$ domain. Developmental Biology 264 255-262.

Kurokawa M, Sato K \& Fissore RA 2004a Mammalian fertilization: from sperm factor to phospholipase Czeta. Biology of the Cell 96 37-45.

Kurokawa M, Sato K, Smyth J, Wu H, Fukami K, Takenawa T \& Fissore RA 2004 $b$ Evidence that activation of Src family kinase is not required for fertilization-associated $\left[\mathrm{Ca}^{2+}\right] i$ oscillations in mouse eggs. Reproduction 127 441-454.

Livingston BT, VanWinkle CE \& Kinsey WH 1998 Protein tyrosine kinase activity following fertilization is required to complete gastrulation, but not for initial differentiation of endoderm and mesoderm in the sea urchin embryo. Developmental Biology 193 90-99.

Mehlmann LM \& Jaffe LA 2005 SH2 domain-mediated activation of an SRC family kinase is not required to initiate $\mathrm{Ca}^{2+}$ release at fertilization in mouse eggs. Reproduction 129 557-564. 
Moore KL \& Kinsey WH 1995 Effects of protein tyrosine kinase inhibitors on egg activation and fertilization-dependent protein tyrosine kinase activity. Developmental Biology 168 1-10.

Muslin AJ, Klippel A \& Williams LT 1993 Phosphatidylinositol 3-kinase activity is important for progesterone-induced Xenopus oocyte maturation. Molecular and Cellular Biology 13 6661-6666.

Nakagata N 1992 Production of normal young following insemination of frozen-thawed mouse spermatozoa into fallopian tubes of pseudopregnant females. Jikken Dobutsu 41 519-522.

Roche S, McGlade J, Jones M, Gish GD, Pawson T \& Courtneidge SA 1995 Requirement for Src family protein tyrosine kinases in G2 for fibroblast cell division. Science 269 1567-1569.

Runft LL, Watras J \& Jaffe LA 1999 Calcium release at fertilization of Xenopus eggs requires type I IP(3) receptors, but not SH2 domainmediated activation of PLCgamma or G(q)-mediated activation of PLCbeta. Developmental Biology 214 399-411.

Runft LL, Jaffe LA \& Mehlmann LM 2002 Egg activation at fertilization: where it all begins. Developmental Biology 245 237-254.

Sakakibara K, Sato K, Yoshino K, Oshiro N, Hirahara S, Mahbub Hasan AK, Iwasaki T, Ueda Y, Iwao Y, Yonezawa K \& Fukami Y 2005 Molecular identification and characterization of Xenopus egg uroplakin III, an egg raft-associated transmembrane protein that is tyrosine-phosphorylated upon fertilization. Journal of Biological Chemistry 280 15029-15037.

Sato K, Tokmakov AA \& Fukami Y 2000a Fertilization signaling and protein-tyrosine kinases. Comparative Biochemistry and Physiology 126 129-148.

Sato K, Tokmakov AA, Iwasaki T \& Fukami Y 2000b Tyrosine kinasedependent activation of phospholipase Cgamma is required for calcium transient in Xenopus egg fertilization. Developmental Biology 224 453-469.

Sette C, Paronetto MP, Barchi M, Bevilacqua A, Geremia R \& Rossi P 2002 Tr-kit-induced resumption of the cell cycle in mouse eggs requires activation of a Src-like kinase. EMBO Journal 21 5386-5395.

Sharma D, Holets L, Zhang X \& Kinsey WH 2005 Role of Fyn kinase in signaling associated with epiboly during zebrafish development Developmental Biology 285 462-476.

Summers MC, Bhatnagar PR, Lawitts JA \& Biggers JD 1995 Fertilization in vitro of mouse ova from inbred and outbred strains: complete preimplantation development in glucose-supplimented KSOM. Biology of Reproduction 53 431-437.
Superti-Furga G \& Courtneidge SA 1995 Structure-function relationships in Src-family and related protein tyrosine kinases. BioEssays 17 321-330.

Talmor A, Kinsey WH \& Shalgi R 1998 Expression and immunolocalization of p59c-fyn tyrosine kinase in rat eggs. Developmental Biology 194 38-46.

Talmor-Cohen A, Tomashov-Matar R, Eliyahu E, Shapiro R \& Shalgi R 2004a Are Src family kinases involved in cell cycle resumption in rat eggs? Reproduction 127 455-463.

Talmor-Cohen A, Tomashov-Matar R, Tsai WB, Kinsey WH \& Shalgi R $2004 b$ Fyn kinase-tubulin interaction during meiosis of rat eggs. Reproduction 128 387-393.

Wong L, Lieser S, Chie-Leon B, Miyashita O, Aubol B, Shaffer J, Onuchic JN, Jennings PA, Woods VL Jr \& Adams JA 2004 Dynamic coupling between the $\mathrm{SH} 2$ domain and active site of the $\mathrm{COOH}$ terminal Src kinase, Csk. Journal of Molecular Biology 341 93-106.

Wright SJ \& Schatten G 1995 Protein tyrosine phosphorylation during sea urchin fertilization: microtubule dynamics require tyrosine kinase activity. Cell Motility and the Cytoskeleton 30 1122-1135.

Wu W \& Kinsey WH 2001 Fertilization triggers activation of Fyn kinase in the zebrafish egg. International Journal of Developmental Biology 44 837-841.

Wu X, Viveiros MM, Eppig JJ, Bai Y, Fitzpatrick SL \& Matzuk MM 2003 Zygote arrest 1 (Zar1) is a novel maternal-effect gene critical for the oocyte-to-embryo transition. Nature Genetics 33 187-191.

Zheng XM, Resnick RJ \& Shalloway D 2000 A phosphotyrosine displacement mechanism for activation of Src by PTPa. EMBO Journal 19 964-978.

Zheng XM, Resnick RJ \& Shalloway D 2002 Mitotic activation of protein-tyrosine phosphatase alpha and regulation of its Srcmediated transforming activity by its sites of protein kinase C phosphorylation. Journal of Biological Chemistry 277 21922-21929.

Received 20 February 2006

First decision 31 March 2006

Revised manuscript received 23 May 2006

Accepted 1 June 2006 